\title{
COVID 19. LA PANDEMIA, EL DERECHO A LA SALUD EN ECUADOR Y VENEZUELA
}

\section{COVID 19. THE PANDEMIC, THE RIGHT TO HEALTH IN ECUADOR AND VENEZUELA}

\author{
Rosa Angélica Romero Chico ${ }^{1}$ \\ Dante Pino Pascucci Stelluto²
}

Recibido: 2019-12-20 / Revisado: 2020-03-10 / Aceptado: 2020-05-05 / Publicado: 2020-07-01

Forma sugerida de citar: Romero-Chico, R. A. y Pascucci-Stelluto, D. P. (2020). COVID 19. La pandemia, el derecho a la salud en Ecuador y Venezuela. Retos de la Ciencia. 4(9), pp. 46-56. https://doi.org/10.53877/rc.4.9.20200701.05

\section{RESUMEN}

El objeto de estudio en el trabajo es el estado del derecho a la salud de la población ecuatoriana y venezolana afectada por la enfermedad de la COVID 19. El objetivo es, realizar un proceso de revisión del tratamiento jurídico dado en Ecuador y en Venezuela, siendo el marco legal diferente en la aplicación empresarial puesto que en Ecuador se contempla la gestión específica a través del área de seguridad laboral y salud en el trabajo. Dentro del análisis del derecho comparado, método aplicado en este estudio en el cual se hace interpretación hermenéutica de textos legales, se evidencia que las Constituciones de ambos países son parecidas en tanto que corresponden al tipo de Constituciones garantistas de los derechos humanos, particularmente los de carácter social, mientras que los modelos de manejo y el uso de los medios de información y comunicación son radicalmente diferentes.

Palabras claves: derecho, derechos humanos salud, pandemia, salud ocupacional

\section{ABSTRACT}

The object of study at work is the state of the right to health of the Ecuadorian and Venezuelan population affected by the COVID 19 disease. The objective is to carry out a review process of the legal treatment given in Ecuador and Venezuela, being the different legal framework in the business application since in Ecuador specific

\footnotetext{
${ }_{1}^{1}$ Magíster en Desarrollo Humano. Profesora de la Facultad de Psicología de la Universidad Central del Ecuador. E-mail: raromeroch@uce.edu.ec / https://orcid.org/0000-0002-8311-5035

2 Ph.D en Gerencia Avanzada. Profesor titular e Investigador de la Universidad De Los Andes. Venezuela. E-mail: dante@ula.edu.ve / https://orcid.org/0000-0003-3262-6174
} 
management is contemplated through the area of occupational safety and health at work. Within the analysis of comparative law, a method applied in this study in which hermeneutical interpretation of legal texts is made, it is evident that the Constitutions of both countries are similar insofar as they correspond to the type of Constitutions that guarantee human rights, particularly those of social character, while the management models and the use of the information and communication media are radicall.

Keywords: law, human rights health, pandemic, occupational health.

\section{INTRODUCCIÓN.}

El objeto del presente estudio es el estado del derecho a la salud de la población en Ecuador y en Venezuela que se encuentra confrontando la enfermedad infecciosa COVID 19. De allí que esta investigación tiene como finalidad comparar el tratamiento jurídico del derecho a la salud en Ecuador y Venezuela, al tiempo que lo identifica y expone. Sin duda que existe la necesidad de conocer y entender, en ambos países, el derecho a la salud de la población durante la pandemia, de allí la importancia de la exposición y análisis que deriva del derecho comparado en lo que concierne a las realidades ecuatoriana y venezolana. Del uso de ese tipo de análisis surge la posibilidad de mejorar los resultados de cara a las estrategias que se utilicen para enfrentar la epidemia en un futuro mediato y así aportar acciones específicas, tanto en forma individual como social, desde esta área del conocimiento.

Este estudio contiene datos estadísticos relativos al impacto del Covid 19, tanto en Ecuador como en Venezuela. Igualmente, se da a conocer lo que resulta de la revisión, estudio y análisis documental de lo normativo jurídico como garantía del Derecho Humano a la salud, así como la documentación oficial y aquellos estudios hechos en el terreno que son dados a conocer mediante diversas publicaciones.

Esta investigación documental, como ha sido dicho, además de lo descriptivo, utiliza el método normativo dado que alude a la importancia de normas y valores, esto es, a los principios esenciales al deber ser. El tema es tratado a la luz de una razón epistémica crítica que estudia y considera lo planteado en la dimensión terrena y con las herramientas que aporta la tríada dialéctica a los fines de abordar una realidad que forma parte de una totalidad que muestra su complejidad.

\section{DESARROLLO}

Por efecto de la pandemia y de los diversos conflictos de la estructura económica y superestructura social existentes tanto en Ecuador como en Venezuela, la situación de salud en ambos países se torna crítica. Cualquier solución requerirá de inteligencia, requerirá de la aplicación de estrategias desde el paradigma de la Gerencia Avanzada para cumplir con los derechos, y mejorar las condiciones de vida. 
La salud, por tanto, la vida está amenazada, hecho que resulta público, notorio y comunicacional, además, su rápida propagación "produce cuadros clínicos que van desde el resfriado común hasta enfermedades más graves" (MSP, 2020).

Vale decir que, de cara a la pandemia existente por el coronavirus, en Ecuador y Venezuela, como en muchos otros países, se aprecian conductas que van desde políticas de confinamiento aplicadas por las autoridades públicas, políticas de prevención, políticas de reincorporación socio económica, así como actitudes extremistas en algunos sectores afines a las tesis del negacionismo, hasta teorías del complot o de la conspiración en un ambiente de desconfianza y de descalificación.

Las autoridades del Poder Ejecutivo de cada país hacen alocuciones diarias dando cuenta de lo que es su política en al área sanitaria asistencial; el discurso oficial es más de carácter propagandístico que real, pues se observa que médicos, enfermeras y todo el equipo de salud que se encuentra en la primera línea de trabajo para atender los casos de personas afectadas por el virus, dan cuenta de graves carencias. Falta de insumos y condiciones intrahospitalarias inapropiadas, con medidas de bioseguridad muy deficientes, forman parte de las dificultades que el personal de salud debe enfrentar.

Mientras el "conflicto de Venezuela se ha transformado en una crisis con implicaciones no sólo locales o nacionales, sino también de alcance regional latinoamericano, continental" (Elcano, 2019), en Ecuador se suma el cierre de contratos ocasionales y falta de consignación de las remuneraciones en el sector público de la salud, despidos en la empresa privada.

Las cifras oficiales, hasta el 10 de septiembre 2020, comunican la siguiente situación de la salud frente a la pandemia, dentro de un medio socio político que evidencia "a la prensa independiente en Venezuela, que tiene problemas para importar papel periódico, es comprada por empresas anónimas cercanas al gobierno y tiene a muchos de sus periodistas enjuiciados o en la cárcel misma" $(B B C, 2016)$ y donde el líder de la asamblea nacional "Juan Guaidó, ascendió al Olimpo de la alicaída política venezolana con su (auto) proclamación como presidente encargado" (NUSO, 2019):

Tabla 1.

Cifras oficiales comunicadas en cada país sobre la salud frente a la pandemia

\begin{tabular}{|l|l|l|}
\hline Datos Por País & Venezuela & Ecuador \\
\hline Fecha & 10 sept 2020 & 10 sept 2020 \\
\hline Fuente & $\begin{array}{l}\text { Ministerio del Poder } \\
\text { Popular para la } \\
\text { Salud/Blog Patria (Patria } \\
\text { Blog, 2020) }\end{array}$ & $\begin{array}{l}\text { Ministerio de Salud } \\
\text { Pública/Diario El } \\
\text { Universo (Mena \& } \\
\text { Serrano,2020) }\end{array}$ \\
\hline Casos confirmados & 58.663 & 113206 \\
\hline Casos descartados & sin dato & 168714 \\
\hline Casos recuperados: & 46.960 & $\begin{array}{l}\text { al 6/sept/2020: 91 242 } \\
\text { (corresponden al 83\% de } \\
\text { casos confirmados). }\end{array}$ \\
\hline
\end{tabular}




\begin{tabular}{|l|l|l|}
\hline Fallecidos: & $\begin{array}{l}468 \text { (de estos hasta el 8 } \\
\text { sept, 100 médicos, } \\
\text { medio El Diario) (Ramos, } \\
\text { 2020); 155 médicos es el } \\
\text { número dado por La } \\
\text { Federación Médica de } \\
\text { Venezuela (FMV), } \\
\text { gremio profesional que } \\
\text { agrupa a los médicos } \\
\text { venezolanos. }\end{array}$ & $\begin{array}{l}\text { confirmados y } 3778 \\
\text { probables. }\end{array}$ \\
& $\begin{array}{l}\text { sin dato } \\
\text { Muestras tomadas }\end{array}$ & $\begin{array}{l}343 \text { 624, de las } \\
\text { cuales 61 704 están } \\
\text { represadas, sin resultado }\end{array}$ \\
\hline $\begin{array}{l}\text { Nota: } \\
\text { * Nohemi Ramos, parte de la directiva de Médicos Unidos por Venezuela (MUV), } \\
\text { indica el retraso en los reportes, los datos de los fallecimientos solo son los } \\
\text { totalmente confirmados por familiares o personal sanitario. }\end{array}$
\end{tabular}

Fuente: Elaboración propia en base a las fuentes citadas

En Venezuela hay una clasificación de los casos de COVID 19 según el lugar de origen del contagio: casos endógenos o comunitarios y casos exógenos o importados; respecto a estos últimos Belchi (2020) ha señalado en trabajo publicado que los migrantes venezolanos regresan a su país en razón de la discriminación por COVID19 (2020), se indica que son muchas las quejas en cuanto al trato que han recibido los pacientes, toda vez que estos son venezolanos de la diáspora que regresan del exterior por la crisis que viven en los países que los acogieron: pérdida de trabajo, desalojo por mora en el pago al día de los cánones de arrendamiento de los inmuebles en los que se encontraban en calidad de arrendatarios (en no pocos casos desalojos violentos y forzados), así como, en ciertas ocasiones, manifestaciones de xenofobia. Del trabajo aludido se infiere que estas personas, no tienen otra opción distinta a la de regresar a Venezuela en espera del apoyo gubernamental venezolano en funciones o del gobierno virtual, el que encarna uno de los diputados de la oposición más radicalizada. Ambos poderes en pugna manejan recursos.

A la crítica situación política que desde hace varios años está presente en el país venezolano, se suman las graves dificultades económicas y sociales que se traducen en escasez de electricidad, de agua, de gasolina, de gas y de transporte; deterioro general del aparato productivo con el inevitable cierre de empresas, pérdidas de puestos de trabajo, precaria producción agropecuaria, poca movilidad social, empobrecimiento generalizado, agobiante hiperinflación y caída sostenida del producto interno bruto (PIB); la crisis en la educación y en el sector salud y la pandemia, así como un bloqueo internacional de parte del gobierno de los Estados Unidos de Norteamérica, entre otros problemas, hacen sumamente complicada la vida del pueblo venezolano. Ecuador en base al nivel de toma de decisiones, inversiones, gestión en políticas públicas y la destructiva pandemia, va camino hacia una dolorosa migración; son latentes y difíciles sus sendas socioeconómicas que se 
reflejan en: el desempleo, alto costo de la vida, cierre de empresas, incremento de la delincuencia y narcotráfico, menor apoyo presupuestario para salud y educación, mayor empobrecimiento evidenciado a través de deudas a organismos internacionales y países dominantes en el mercado global. La segunda jornada de la 24 ${ }^{a}$ Conferencia anual de la CAF-Banco de Desarrollo de América Latina, celebrada del 09 al 11 de septiembre de 2020, se ocupó de tratar y debatir la situación que confrontan los sistemas de salud en la región y, de modo muy especial, la situación que está viviendo la población con mayor riesgo, la de mayor vulnerabilidad de cara a la crisis sanitaria y a los demás aspectos sociales, económicos y políticos.

Tabla 2.

Análisis comparativo de la política gubernamental, acerca de la realización de pruebas diagnósticas frente a la pandemia

\begin{tabular}{|l|l|l|}
\hline Datos por país & Venezuela & Ecuador \\
\hline Fuente & $\begin{array}{l}\text { BBC Mundo, Ut supra } \\
\text { (Olmo, 2020). }\end{array}$ & $\begin{array}{l}\text { El Comercio, 2 julio 2020 } \\
\text { (Heredia, 2020). }\end{array}$ \\
\hline Control de la pandemia & $\begin{array}{l}\text { Pruebas diagnósticas } \\
\text { COVID 19. }\end{array}$ & $\begin{array}{l}\text { ¿Cuáles son los } \\
\text { requisitos para hacerse } \\
\text { una prueba de covid-19 } \\
\text { El gobierno solo ha } \\
\text { autorizado la realización } \\
\text { de las pruebas } \\
\text { diagnósticas al Instituto } \\
\text { Nacional de Higiene, } \\
\text { ubicado en Caracas, lo } \\
\text { que, según expertos } \\
\text { médicos limita la } \\
\text { capacidad de detectar la } \\
\text { penetración del virus en } \\
\text { territorio venezolano. No } \\
\text { autoriza la realización de } \\
\text { las pruebas a las } \\
\text { universidades y clínicas } \\
\text { privadas }\end{array}$ \\
\hline
\end{tabular}

Fuente: Elaboración propia en base a las fuentes mencionadas 
Tabla 3.

Posición comparativa del Índice de Seguridad Sanitaria Mundial, de la Universidad Johns Hopkins.

\begin{tabular}{|l|l|l|}
\hline $\begin{array}{l}\text { Puesto del país en la } \\
\text { respuesta rápida y } \\
\text { mitigación de la } \\
\text { propagación de la COVID } \\
19\end{array}$ & $\begin{array}{l}\text { 180; puntuación 19,7 } \\
\text { (siendo el puesto 1 Reino } \\
\text { Unido, puesto 2 Estados } \\
\text { Unidos, puesto 3 Suiza } \\
\text { en otros). }\end{array}$ & $\begin{array}{l}\text { 85, puntuación 39,5 } \\
\text { (siendo el puesto 1 Reino } \\
\text { Unido, puesto 2 Estados } \\
\text { Unidos, puesto 3 Suiza } \\
\text { en otros). }\end{array}$ \\
\hline
\end{tabular}

Fuente: Elaboración propia en base a la información de GHSINDEX, (Center for Health Security, 2019).

La respuesta nacional ecuatoriana, se evidencia principalmente a través de gestión desde las entidades de control, siendo la principal el Comité de Operaciones de Emergencia Nacional (COE, 2020) a través del cual se ha organizado el accionar desde las diferentes áreas como:

En la salud: declarando el estado de emergencia sanitaria a nivel nacional, uso de pruebas rápidas para la detección de anticuerpos, disposiciones de bioseguridad como distanciamiento social, uso de mascarilla y lavado permanente de manos.

En la seguridad territorial: declarando el aislamiento preventivo obligatorio por un período ininterrumpido de catorce (14) días a todo viajero sin importar la nacionalidad, las restricciones de movilidad y aplicación de salvoconductos; semaforización, en base de indicadores epidemiológicos y conducta ciudadana; generación de alertas a través del Comité de Operaciones de Emergencia Nacional (COE) para la toma de decisiones, a través de las mesas técnicas y grupos de trabajo.

En lo laboral: declarando el teletrabajo emergente; la aplicación de la reducción, modificación o suspensión emergente de la jornada laboral durante la declaratoria de emergencia sanitaria, posibilidad de terminación del contrato individual de trabajo de conformidad con la causal 6 del artículo 169 del Código de Trabajo, declaración que el COVID19 no constituye un accidente de trabajo ni una enfermedad profesional. Que las unidades o departamentos de seguridad y salud internos determinen las personas que de acuerdo con su valoración y al riesgo de contagio en las actividades que ejecutan, puedan asistir de manera presencial. Además, será obligación de los trabajadores acatar todas las normas y procedimientos de prevención establecidos por el empleador.

En lo económico: determinar directrices para la reactivación económica a través del retorno progresivo al trabajo primero virtual, semipresencial luego presencial. Objetivo al cual coopera el mérito del Sello Internacional Safe Travel, recibido por el alcalde de Quito en septiembre 2020 "otorgado por el Consejo Mundial de Viajes y Turismo (WTTC), en reconocimiento a la aplicación de los protocolos de bioseguridad para la prevención de la COVID-19 en la actividad turística" (La República, 2020), solo ochenta (80) países en el mundo tienen este sello.

Medidas parecidas han sido tomadas en la hermana República Bolivariana de Venezuela. 
En lo legal, colijamos que el derecho a la salud es un derecho básico, que en esta pandemia no se ha podido cubrir a cabalidad debido a las condiciones socio económicas y políticas, aún, cuando en las leyes supremas ecuatoriana y venezolana se encuentra claramente determinado, así: Artículo 32 de la Constitución de la República del Ecuador del 2011 (Constitución, 2011):

"La salud es un derecho que garantiza el Estado, cuya realización se vincula al ejercicio de otros derechos, entre ellos el derecho al agua, la alimentación, la educación, la cultura física, el trabajo, la seguridad social, los ambientes sanos y otros que sustentan el buen vivir. El Estado garantizará este derecho mediante políticas económicas, sociales, culturales, educativas y ambientales; y el acceso permanente, oportuno y sin exclusión a programas, acciones y servicios de promoción y atención integral de salud, salud sexual y salud reproductiva. La prestación de los servicios de salud se regirá por los principios de equidad, universalidad, solidaridad, interculturalidad, calidad, eficiencia, eficacia, precaución y bioética, con enfoque de género y generacional".

Analizando la Constitución venezolana, artículo 83 (Constitución, 1999) se afirma que:

"La salud es un derecho social fundamental, [...] Todas las personas tienen derecho a la protección de la salud. Así como el deber de participar activamente en su promoción y defensas, y el de cumplir con las medidas sanitarias y de saneamiento que establezca la Ley de conformidad con los tratados y convenios internacionales suscritos y ratificados por la República".

El derecho a la salud se encuentra consagrado en la Declaración Universal de los Derechos Humanos (ONU, 2015). El derecho a la vida ... que asegure, [...] asistencia médica y servicios sociales necesarios e indispensables. Puede apreciarse el carácter integral e interactuante que tiene este derecho.

Desde la salud ocupacional se genera un "plan de emergencia para continuar las actividades, identificación de recursos técnicos del trabajador, el establecimiento de canales de comunicación individualizados y/o de grupo..." (INNST MITES, 2020), que permitan mitigar los riesgos psicosociales en el trabajo a distancia por confinamiento físico y presencial por la pandemia, como: "estrés, fatiga mental, alteración en los hábitos de sueño" (INNST, 2020), alteración en la rutina de ejercicio físico, condiciones de trabajo diferentes horarios complejos, pudiendo producir a largo plazo enfermedades como "cardiovasculares, respiratorias, inmunitarias, gastrointestinales, dermatológicas, endocrinológicas, musculoesqueléticas y mentales" (ISTAS, 2020).

\section{METODOLOGÍA}

El artículo ofrece la revisión de documentos legales y de interés público que esclarecen el derecho a la salud. Los métodos utilizados en su desarrollo son:

Método de análisis tratado como derecho comparado, luego de leer y analizar los documentos se ha identificado coincidencias, diferencias en las constituciones y leyes de los países respecto al derecho a la salud. 
Método deductivo, habiendo leído documentos generales como son las Constituciones respectivas, se ha revisado las leyes de cada realidad, las políticas establecidas y sus aplicaciones en calidad de evidencias o hechos de inclusión de cada país en cuestión. Es decir, el sentido de la gestión es, desde lo general a lo particular.

Método comparativo: permite contrastar dos realidades legales bajo condiciones diferentes, y con culturas semejantes por ser parte del mismo segmento geográfico. El propósito de su uso es determinar aquellos aspectos que se asemejan y los elementos diferenciadores.

\section{RESULTADOS Y DISCUSIÓN}

Dentro del análisis del derecho comparado se evidencia que como constituciones son parecidas; mientras que los modelos de manejo o enfrentamiento a la pandemia, el uso de los medios de información y comunicación son radicalmente diferentes. Ecuador presenta un manejo de datos e información a la población más abierto que Venezuela. La falta de precisión en la información, medios para el desarrollo tecnológicos de las soluciones de las dos repúblicas es una semejanza.

Siendo naciones hermanas el marco legal varía en la aplicación institucional, empresarial, puesto que en Ecuador se contempla la gestión específica a través del área de seguridad laboral y salud en el trabajo, mientras que por el modelo venezolano el accionar empresarial no presenta evidencia.

En Ecuador existe un proyecto de Ley Orgánica se Seguridad y Salud Ocupacional, esta categoría de orgánica va a impactar en el avance y cumplimiento de lo dispuesto en el área, en caso de ser aprobada. La evidencia de la realidad ecuatoriana es que la normativa data entre tres y cuatro décadas, que no se moviliza con la dinámica tecnológica, lo que permite colegir y compartir que existe una verdadera oportunidad de mejora técnica y profesional. En Venezuela no existe evidencia sobre una ley orgánica parecida, ni normativa actualizada.

En el período de pandemia en las organizaciones en general especialmente en las privadas, el área que ha asumido la responsabilidad preventiva es el área de Seguridad en el Trabajo y Salud Ocupacional. Junto con la alta gerencia, genera un modelo de gestión que tiene como norte mantener la salud de los trabajadores y generar recursos para mantener una organización productiva.

La salud como derecho humano se encuentra dentro de la planificación y gestión del modelo político y social ecuatoriano, antes más que ahora. Mientras que la planificación de salud no evidencia la gestión y condiciones de la población de la república bolivariana, "describir la situación actual de Venezuela es tan difícil como lograr que aquellos que no la viven en carne propia la comprendan" (Consolata, 2017l), hecho suscitado incluso antes de la presencia de la pandemia, al punto de que su población migra activamente hacia otros países. 
La realidad pandémica genera un escenario reactivo para las repúblicas mencionadas, así como la atención, la medición y estrategias para el diagnóstico de la salud del trabajador.

\section{CONCLUSIONES}

La revisión del derecho a la salud en el período de pandemia, renova el pensamiento, estimula el accionar para generar desde el rol ciudadano las condiciones favorables requeridas.

La revisión de la salud como derecho humano, expone a la comunidad científica y social hechos que incrementan el nivel de conocimiento y un reflejo de la realidad más objetiva. Coligiendo así que, a más información recibida mayor será la calidad del razonamiento individual y participación frente a su escenario social.

La ardua tarea de complementar la información desde el derecho, la psicología (SESO) y la salud pública, busca ofrecer una visión más compleja y útil para la toma de decisiones, desde los diferentes campos de los lectores.

Dentro del campo productivo, los dos países evidencian la necesidad de una legislación actualizada, que norme el accionar preventivo en salud pública, en seguridad y salud ocupacional.

\section{REFERENCIAS BIBLIOGRÁFICAS}

Asamblea Nacional (2010). Ley Orgánica de Educación Superior. Capítulo Principio de Pertinencia. Distrito Metropolitano de Quito.: Gaceta Judicial. Website: https://www.siteal.iiep.unesco.org/sites/default/files/sit_accion_files/ec_6011.p df

Asamblea Nacional (2018). Propuesta de Proyecto de Ley Orgánica de Seguridad y Salud en el Trabajo. Distrito Metropolitano de Quito: Gaceta Judicial.

Website: https://observatoriolegislativo.ec/legislacion/proyectos-de-ley/proyecto-deley-organica-de-seguridad-y-salud-en-el-trabajo_28172

Pardo, D (2016). 5 mitos sobre la crisis en Venezuela (y lo que pasa en realidad). BBC Mundo. 21 de julio 2016. Website: https://www.bbc.com/mundo/noticiasamerica-latina-36837574

Belchi, A. (2020). Advierten que migrantes venezolanos regresan a su país ante discriminación por COVID-19. Voanoticias, 10 de septiembre de 2020. Website: https://www.voanoticias.com/venezuela/advierten-regresomigrantes-venezolanos-discriminados-covid-19

CAF. Banco de Desarrollo de América Latina (2020). 24 conferencia Anual CAF. Respuesta de CAF frente al COVID-19. Website: https://www.caf.com/es/actualidad/eventos/2020/09/24-conferencia-anual-caf/

Center for Health Security (2019). Global Health Security Index. Estados Unidos: GHS Index 2019. Website: https://www.ghsindex.org/wpcontent/uploads/2019/10/2019-Global-Health-Security-Index.pdf 
COE (2020). Resoluciones COE. Ecuador: Secretaria Nacional de Gestión de Riesgos. Website: https://www.gestionderiesgos.gob.ec/resoluciones-coe/ https://www.gestionderiesgos.gob.ec/resoluciones-coe-nacional-11-deseptiembre-2020/

Congreso Nacional (2005). Código de trabajo. Capítulo I. Determinación de los riesgos y de la responsabilidad del empleador. Distrito Metropolitano de Quito: Gaceta Judicial.

Otieno, P (2017). Venezuela y su Realidad Actual: Sálvese quien pueda. Italia: Consolata.Website: https://www.consolata.org/new/index.php/mission/nostridicono/item/1404venezuela-y-su-realidad-actual-salvese-quien-pueda

Constitución (1999). Constitución de la República Bolivariana de Venezuela. Venezuela: Ministerio del Poder Popular para la comunicación y la información de Gobierno.

Website: http://www.minci.gob.ve/wpcontent/uploads/2011/04/CONSTITUCION.pdf

Constitución (2011). Constitución de la República del Ecuador. Ecuador: Organización de los Estados Americanos. Website: https://www.oas.org/juridico/pdfs/mesicic4_ecu_const.pdf

Heredia, V. (2 de julio 2020). ¿Cuáles son los requisitos para hacerse una prueba de covid-19 en Quito? Quito: El Comercio.com Website: https://www.elcomercio.com/actualidad/laboratorio-requisitos-pruebascoronavirus-quito.html

INNST MITES (2020). Prevención de Riesgos psicosociales en situación de trabajo a distancia debida al COVID 19. Recomendaciones para el empleador. España: Instituto Nacional de Seguridad y Salud en el Trabajo. Website: https://www.insst.es/documentacion/catalogo-de-publicaciones/riesgospsicosociales-y-trabajo-a-distancia-por-covid-19.-recomendaciones-para-elempleado

ISTAS (2020). Riesgos Psicosociales. España: Instituto Sindical de Trabajo, Ambiente y Salud. Website: https://istas.net/salud-laboral/peligros-y-riesgoslaborales/riesgossicosociales\#: :text=Los\%20riesgos\%20psicosociales\%20perjudican\%20la,\% 2C\%20endocrinol\%C3\%B3gicas\%2C\%20musculoesquel\%C3\%A9ticas\%20y $\% 20$ mentales.

La República (2020). Periodismo Digital, Quito recibe el sello internacional de Safe travel. Ecuador: La República EC. Website: https://www.larepublica.ec/blog/2020/09/17/quito-recibe-el-sello-internacionalde-safe-travel/

Malamud, C et al (2019). La crisis de Venezuela y el tablero geopolítico internacional. Real Instituto Elcano. Website: http://www.realinstitutoelcano.org/wps/portal/rielcano_es/contenido?WCM_GL OBAL_CONTEXT=/elcano/elcano_es/zonas_es/ari25-2019-malamud-nunezcrisis-venezuela-tablero-geopolitico-internacional 
Mena, $P$ et al (2020). Casos de coronavirus en Ecuador, al jueves 10 de septiembre: 113206 confirmados y 10749 fallecidos. Quito: El Universo.com Website: https://www.eluniverso.com/noticias/2020/09/10/nota/7972585/coronaviruscovid19-ecuador-casos-contagios-muertes-10-septiembre

MSP (2020). Coronavirus. 2019. Quito: Ministerio de Salud Pública. Website: https://www.salud.gob.ec/coronavirus-covid-19/

Olmo, G. (2020). Coronavirus en Venezuela: los informes que advierten que la pandemia es una bomba de tiempo en el país sudamericano. Venezuela: BBC News Mundo en Venezuela. Website: https://www.bbc.com/mundo/noticiasamerica-latina-52815754

ONU (2015). Declaración Universal de los Derechos Humanos. Organización de las Naciones Unidas. Website: https://www.un.org/es/documents/udhr/UDHR_booklet_SP_web.pdf

Patria Blog (2020). Estadísticas Venezuela. Venezuela: Patria Blog, Vicepresidencia Ejecutiva de la República Bolivariana de Venezuela. Comisión Presidencial para el Control y la Prevención de la COVID-19. Website: https://covid19.patria.org.ve/estadisticas-venezuela/

Ramos, N (2020). 100 médicos han perdido la vida por COVID-19 en Venezuela. En análisis, especial de Jackelin Díaz. Venezuela: El Diario.Com Website: https://Eldiario.Com/2020/08/27/Medicos-Muertos-Coronavirus-Venezuela/

SENPLADES (2017). Plan Nacional De Desarrollo 2017-2021 Toda una Vida. Distrito Metropolitano de Quito: Consejo Nacional de Planificación. Website: https://www.planificacion.gob.ec/wpcontent/uploads/downloads/2017/10/PNBV-26-OCT-

FINAL_OK.compressed1.pdf

Shuterland, M (2019). Venezuela: ¿por qué volvió a fracasar la oposición? NUSO.org. Website: $\quad$ https://nuso.org/articulo/venezuela-por-que-volvio-fracasar-laoposicion/ 